\title{
Weakly Complementary Cycles in 3-Connected Multipartite Tournaments
}

\author{
Lutz Volkmann And STEFAn Winzen \\ Lehrstuhl II für Mathematik, RWTH Aachen, 52056 Aachen, Germany \\ e-mail : volkm@math2.rwth-aachen.de and winzen@math2.rwth-aachen.de
}

\begin{abstract}
The vertex set of a digraph $D$ is denoted by $V(D)$. A $c$-partite tournament is an orientation of a complete $c$-partite graph. A digraph $D$ is called cycle complementary if there exist two vertex disjoint cycles $C_{1}$ and $C_{2}$ such that $V(D)=V\left(C_{1}\right) \cup V\left(C_{2}\right)$, and a multipartite tournament $D$ is called weakly cycle complementary if there exist two vertex disjoint cycles $C_{1}$ and $C_{2}$ such that $V\left(C_{1}\right) \cup V\left(C_{2}\right)$ contains vertices of all partite sets of $D$. The problem of complementary cycles in 2-connected tournaments was completely solved by Reid [4] in 1985 and Z. Song [5] in 1993. They proved that every 2-connected tournament $T$ on at least 8 vertices has complementary cycles of length $t$ and $|V(T)|-t$ for all $3 \leq t \leq|V(T)| / 2$. Recently, Volkmann [8] proved that each regular multipartite tournament $D$ of order $|V(D)| \geq 8$ is cycle complementary. In this article, we analyze multipartite tournaments that are weakly cycle complementary. Especially, we will characterize all 3-connected $c$-partite tournaments with $c \geq 3$ that are weakly cycle complementary.
\end{abstract}

\section{Terminology}

In this paper all digraphs are finite without loops and multiple arcs. The vertex set and the arc set of a digraph $D$ are denoted by $V(D)$ and $E(D)$, respectively. If $x y$ is an arc of a digraph $D$, then we write $x \rightarrow y$ and say $x$ dominates $y$, and if $X$ and $Y$ are two disjoint vertex sets or subdigraphs of $D$ such that every vertex of $X$ dominates every vertex of $Y$, then we say that $X$ dominates $Y$, denoted by $X \rightarrow Y$. Furthermore, $X \leadsto Y$ denotes the fact that there is no arc leading from $Y$ to $X$.

If $D$ is a digraph, then the out-neighborhood $N_{D}^{+}(x)=N^{+}(x)$ of a vertex $x$ is the set of vertices dominated by $x$ and the in-neighborhood $N_{D}^{-}(x)=N^{-}(x)$ is the set of vertices dominating $x$. Therefore, if the arc $x y \in E(D)$ exists, then $y$ is an outer neighbor of $x$ and $x$ is an inner neighbor of $y$. The numbers $d_{D}^{+}(x)=d^{+}(x)=$ $\left|N^{+}(x)\right|$ and $d_{D}^{-}(x)=d^{-}(x)=\left|N^{-}(x)\right|$ are called the outdegree and the indegree of $x$, respectively. Furthermore, the numbers $\delta_{D}^{+}=\delta^{+}=\min \left\{d^{+}(x) \mid x \in V(D)\right\}$ and $\delta_{D}^{-}=\delta^{-}=\min \left\{d^{-}(x) \mid x \in V(D)\right\}$ are the minimum outdegree and the minimum

Received October 12, 2006.

2000 Mathematics Subject Classification: 05C20 .

Key words and phrases: Multipartite tournaments, weakly cycle complementarity. 
indegree, respectively.

For a vertex set $X$ of $D$, we define $D[X]$ as the subdigraph induced by $X$. If we replace in a digraph $D$ every arc $x y$ by $y x$, then we call the resulting digraph the converse of $D$, denoted by $D^{-1}$.

If we speak of a cycle, then we mean a directed cycle, and a cycle of length $n$ is called an $n$-cycle. The length of a cycle $C$ is denoted by $L(C)$. A digraph $D$ is called pancyclic if it contains cycles of length $n$ for all $n \in\{3,4, \ldots,|V(D)|\}$. If $x \in V(C)(x \in V(P)$, respectively) for a cycle $C$ (a path $P$ ), then we denote the successor of $x$ in the given cycle (path) by $x^{+}$and the predecessor by $x^{-}$. A digraph $D$ is cycle complementary if there exist two vertex-disjoint cycles $C$ and $C^{\prime}$ such that $V(D)=V(C) \cup V\left(C^{\prime}\right)$.

A digraph $D$ is strongly connected or strong if for each pair of vertices $u$ and $v$, there is a path from $u$ to $v$ in $D$. A digraph $D$ with at least $k+1$ vertices is $k$-connected if for any set $A$ of at most $k-1$ vertices, the subdigraph $D-A$ is strong. The connectivity, denoted by $\kappa(D)$, is then defined to be the largest value of $k$ such that $D$ is $k$-connected. If $\kappa(D)=1$ and $x$ is a vertex of $D$ such that $D-x$ is not strong, then we say that $x$ is a cut-vertex of $D$.

A digraph $D$ is called c-partite, if its underlying graph $G$ is $c$-partite. Especially, a c-partite or multipartite tournament is an orientation of a complete $c$ partite graph. A tournament is a $c$-partite tournament with exactly $c$ vertices. If $V_{1}, V_{2}, \cdots, V_{c}$ are the partite sets of a $c$-partite tournament $D$ and the vertex $x$ of $D$ belongs to the partite set $V_{i}$, then we define $V(x)=V_{i}$. If $D$ is a $c$-partite tournament with the partite sets $V_{1}, V_{2}, \ldots, V_{c}$ such that $\left|V_{1}\right| \leq\left|V_{2}\right| \leq \cdots \leq\left|V_{c}\right|$, then $\left|V_{c}\right|=\alpha(D)$ is the independence number of $D$.

\section{Introduction and preliminary results}

There is an extensive literature about the existence of complementary cycles in digraphs. In 1985, Reid investigated 2-connected tournaments. In this class of digraphs he found an example of a 3-connected regular tournament with seven vertices, which is not cycle complementary.

Example 2.1(Reid [4]). Let $T_{7}$ be the 3-regular and 3-connected tournament presented in Figure 1. Then it is well-known that $T_{7}$ doesn't contain a 3 -cycle $C_{3}$ and a 4-cycle $C_{4}$ such that $V\left(T_{7}\right)=V\left(C_{3}\right) \cup V\left(C_{4}\right)$.

The tournament $T_{7}$ is the sole exception of a 2-connected tournament with at least 6 vertices that is not cycle complementary.

Theorem 2.2(Reid [4]). Let $T$ be a 2-connected tournament with at least $n \geq 6$ vertices. Then either $T$ contains a 3 -cycle and an $(n-3)$-cycle which are vertex disjoint or $T$ is the 7-tournament $T_{7}$.

In 1993, Song [5] extended this result.

Theorem 2.3(Song [5]). If $T$ is a 2-connected tournament with at least eight 
vertices, then $T$ contains two complementary cycles of length $t$ and $|V(T)|-t$ for all $3 \leq t \leq|V(T)| / 2$.

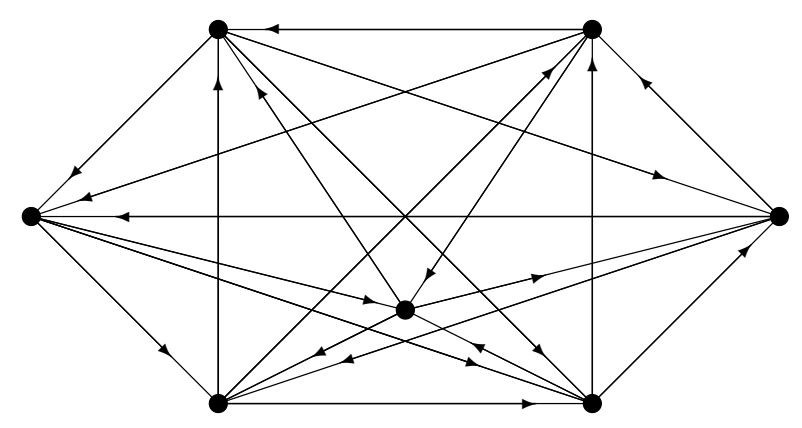

Figure 1: The 3-regular, 3-connected tournament $T_{7}$

The problem of complementary cycles in multipartite tournaments is much more difficult to analyze than in tournaments. This is why up to now only regular multipartite tournaments were considered. Even not all digraphs of this class are cycle complementary as the following example demonstrates.

Example 2.4(Volkmann [8]). Let $V_{1}=\left\{x_{1}, x_{2}\right\}, V_{2}=\left\{y_{1}, y_{2}\right\}$ and $V_{3}=\left\{u_{1}, u_{2}\right\}$ be the partite sets of the 2-connected 3-partite tournament $D_{3,2}$ presented in Figure 2. Then it is a simple matter to verify that $D_{3,2}$ doesn't contain two vertex disjoint cycles.

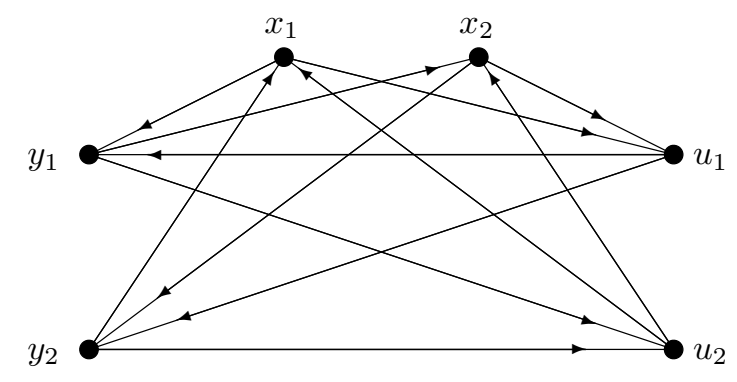

Figure 2: The 2-connected 3-partite tournament $D_{3,2}$

In 2004, Volkmann [8] proved the following result for regular multipartite tournaments.

Theorem 2.5(Volkmann $[8])$. Let $D$ be a regular c-partite tournament. If $c=2$ 
and $|V(D)| \geq 8$ or $c \geq 3$ and $|V(D)| \geq 6$, then $D$ is cycle complementary, unless $D$ is isomorphic to $T_{7}$ in Figure 1 or to $D_{3,2}$ in Figure 2.

This theorem could make believe that the following conjecture of Yeo [11] is valid.

Conjucture 2.6(Yeo [11]). A regular $c$-partite tournament $D$ with $c \geq 4$ has a pair of vertex disjoint cycles of length $t$ and $|V(D)|-t$ for all $t \in\{3,4, \cdots,|V(D)|-3\}$.

In [10], Volkmann showed that this conjecture is valid for $t=3$ with exception of three special digraphs. Moreover, in this article and in [9] he treated the case that $t=4$ in Conjecture 2.6. And in a recent article Korneffel, Meierling, Volkmann and Winzen [3] have shown that Conjecture 2.6 is true for $t=5$.

There is still another unsolved conjecture by Volkmann [7] concerning complementary cycles.

Conjucture 2.7(Volkmann [7]). A multipartite tournament $D$ with $\kappa(D) \geq$ $\alpha(D)+1$ is cycle complementary, unless $D$ is a member of a finite family of multipartite tournaments.

The aim of this article is to weaken the condition that $D$ is cycle complementary in the following way.

Definition 2.8. Let $D$ be a $c$-partite digraph with the partite sets $V_{1}, V_{2}, \cdots, V_{c}$. Two vertex disjoint cycles $C$ and $C^{\prime}$ are called weakly complementary, if they contain vertices of all partite sets of $D$, which means that $\left(V(C) \cup V\left(C^{\prime}\right)\right) \cap V_{i} \neq \emptyset$ for all $1 \leq i \leq c$ and $V(C) \cap V\left(C^{\prime}\right)=\emptyset$. A $c$-partite digraph $D$ with such two cycles is weakly cycle complementary.

Note that a tournament is weakly cycle complementary, if and only if it is cycle complementary. This definition leads to a new problem.

Problem 2.9. Find necessary and/or sufficient conditions for a $c$-partite digraph to be weakly cycle complementary.

Using the weaker Definition 2.8 of cycle complementarity it is possible to characterize $k$-connected multipartite tournaments which are weakly cycle complementary. Especially, in this article we will prove that a 3-strong $c$-partite tournament $D$ with $c \geq 3$ and at least six vertices is weakly cycle complementary unless $D$ is isomorphic to $T_{7}$ in Figure 1 . The following results play an important role to prove this characterization.

Theorem 2.10(Bondy [1]). Each strong c-partite tournament contains an m-cycle for each $m \in\{3,4, \cdots, c\}$.

Theorem 2.11(Goddard, Oellermann [2]). Let D be a strongly connected multipartite tournament with the partite sets $V_{1}, V_{2}, \cdots, V_{c}$. Then every vertex of $D$ belongs to a cycle that contains vertices from exactly $m$ partite sets for each $m \in\{3,4, \cdots, c\}$. 
Corollary 2.12. Every vertex of a strongly connected c-partite tournament $D$ with $c \geq 2$ belongs to a cycle that contains vertices from all $c$ partite sets.

In the following we call a cycle containing the vertex $x_{0}$ and vertices from all partite sets of a multipartite tournament a GodOel-cycle $C\left(x_{0}\right)$.

Theorem 2.13(Tewes, Volkmann [6]). If $D$ is a non-strong c-partite tournament with the partite sets $V_{1}, V_{2}, \cdots, V_{c}$, then there exists a unique decomposition of $V(D)$ into pairwise disjoint subsets $D_{1}, D_{2}, \cdots, D_{p}$, where $D_{i}$ is the vertex set of a strong component of $D$ or $D_{i} \subseteq V_{l}$ for some $l \in\{1,2, \cdots, c\}$ such that $D_{i} \leadsto D_{j}$ for $1 \leq i<j \leq p$ and there are $x_{i} \in D_{i}$ and $x_{i+1} \in D_{i+1}$ such that $x_{i} \rightarrow x_{i+1}$ for $1 \leq i<p$.

\section{Main result}

Theorem 3.1. Let $D$ be a c-partite tournament with $c \geq 3,|V(D)| \geq 6$ and $\kappa(D) \geq 3$. Then $D$ is weakly cycle complementary unless $D$ is isomorphic to $T_{7}$ in Figure 1 .

Proof. Let $D$ be a $c$-partite tournament with $c \geq 3,|V(D)| \geq 6$ and $\kappa(D) \geq 3$. According to Theorem 2.10, $D$ contains a 3 -cycle $C=v_{1} v_{2} v_{3} v_{1}$. Let $D-V(C)$ consist of the partite sets $V_{1}, V_{2}, \cdots, V_{c^{\prime}}$.

First, let $\kappa(D) \geq 4$. In this case $D-V(C)$ is strong and thus it contains a GodOel-cycle $C^{\prime}$. Since $C$ and $C^{\prime}$ are two vertex disjoint cycles in $D$ with vertices from all partite sets, we conclude that $D$ is weakly cycle complementary.

Second, we may assume that $\kappa(D)=3$. If $D-V(C)$ is strong, then as above we see that $D$ is weakly cycle complementary. Hence let $D-\left\{v_{1}, v_{2}, v_{3}\right\}$ be nonstrong. Theorem 2.13 implies that there is a unique decomposition of $V(D)-V(C)$ in subsets $D_{1}, D_{2}, \cdots, D_{p}$, where $D_{i}$ is the vertex set of a strong component of $D-V(C)$ or $D_{i} \subseteq V_{l}$ for some $l \in\left\{1,2, \cdots, c^{\prime}\right\}$ such that $D_{i} \leadsto D_{j}$ for $1 \leq i<j \leq p$ and there are $x_{i} \in D_{i}$ and $x_{i+1} \in D_{i+1}$ such that $x_{i} \rightarrow x_{i+1}$ for $1 \leq i<p$. Suppose that $D$ is not weakly cycle complementary.

If $D_{1}$ is an independent set of vertices and there are vertices $v_{1}^{\prime} \in D_{1}$ and $v_{i} \in V(C)$ such that $v_{1}^{\prime} \leadsto v_{i}$ (without loss of generality, let $i=1$ ), then the fact that $d_{D-\left\{v_{2}, v_{3}\right\}}^{-}\left(v_{1}^{\prime}\right)=0$ yields that $D-\left\{v_{2}, v_{3}\right\}$ is not strong, a contradiction to $\kappa(D)=3$. Hence it remains to treat the case that $V(C) \rightarrow D_{1}$, if $D_{1} \subseteq V_{j}$ for some $1 \leq j \leq c^{\prime}$. Analogously, we see that $D_{p} \rightarrow V(C)$, if $D_{p}$ is an independent set of vertices. If $D_{1}$ is the vertex set of a strong component with $\left|D_{1}\right| \geq 3$, then the fact that $\kappa(D) \geq 3$ implies that there are three pairwise non-incident arcs leading from $V(C)$ to $D_{1}$. Analogously, if $D_{p}$ is the vertex set of a strong component with $\left|D_{p}\right| \geq 3$, then there are three pairwise non-incident arcs leading from $D_{p}$ to $V(C)$.

To prove this theorem we distinguish different cases.

Case 1. Assume that there are at least two vertex sets in $D_{1}, D_{2}, \cdots, D_{p}$ that induce a non-trivial strong component. 
Subcase 1.1. Assume that at least one of these vertex sets is $D_{i}$ with $1<i<p$. Let $x_{1} \in N^{+}\left(v_{1}\right) \cap D_{1}$ and $y_{1} \in N^{-}\left(v_{2}\right) \cap D_{p}$. Let us define $C_{1}=C\left(x_{1}\right)$ if $D\left[D_{1}\right]$ is a non-trivial strong component and $C_{1}=x_{1}$ otherwise. Analogously, let $C_{p}=C\left(y_{1}\right)$ if $D\left[D_{p}\right]$ is a non-trivial strong component and $C_{p}=y_{1}$ otherwise. Similarly, we define $C_{j}(2 \leq j \leq p-1)$ as an arbitrary GodOel-cycle of $D\left[D_{j}\right]$, if $D_{j}$ induces a non-trivial strong component and $C_{j}=v_{j}^{\prime}$ with $v_{j}^{\prime} \in D_{j}$ otherwise. Now it is obvious that $C_{i}$ and $\left(v_{1} C_{1} C_{2} \cdots C_{i-1} C_{i+1} \cdots C_{p} v_{2} v_{3} v_{1}\right.$ or $v_{1} C_{1} C_{2} \cdots C_{i-2} C_{i+1} \cdots C_{p} v_{2} v_{3} v_{1}$ or $\left.v_{1} C_{1} C_{2} \cdots C_{i-1} C_{i+2} \cdots C_{p} v_{2} v_{3} v_{1}\right)$ are two weakly complementary cycles of $D$, if we interpret the second cycle in the following way:

If $D_{1}$ is the vertex set of a non-trivial strong component, then we walk from $v_{1}$ to $x_{1}$ and along the cycle $C_{1}$ until we reach the vertex $x_{1}^{-}$and then we walk to a vertex of $C_{2}$. If however $D_{2}$ is an independent set of vertices such that $v_{2}^{\prime} \in V\left(x_{1}^{-}\right)$ for all $v_{2}^{\prime} \in D_{2}$, then we walk along the cycle $C_{1}$ until $x_{1}^{--}$. In the case that $D_{1}$ is an independent set of vertices we walk from $v_{1}$ to $x_{1}$ and then to a vertex of $D_{2}$.

If we arrive at the vertex $v_{j}^{\prime}$ of a GodOel-cycle $C_{j}$, then we walk along the cycle until we reach the vertex $v_{j}^{\prime-}$ and then we pass over to a vertex of the next component. In the case that the next component is an independent set of vertices that belong to the same partite set as $v_{j}^{\prime-}$ then we stop at the vertex $v_{j}^{\prime--}$ and pass over to the next component.

Finally, if $D_{p}$ induces a non-trivial strong component, then we pay attention that we reach the cycle $C_{p}$ in the vertex $y_{1}^{+}$, then we walk the cycle along until $y_{1}$ and pass over to $v_{2}, v_{3}$ and we finish the cycle with $v_{1}$.

These two cycles lead to a contradiction to our assumption that $D$ is not weakly cycle complementary.

Subcase 1.2. Assume that only the two vertex sets $D_{1}$ and $D_{p}$ induce a non-trivial strong component. Let $v_{1} \rightarrow x_{s}, v_{2} \rightarrow x_{t}$ and $v_{3} \rightarrow x_{m}$ be the three pairwise non-incident arcs leading from $V(C)$ to $D_{1}$. Analogously, let $y_{s} \rightarrow v_{1}, y_{t} \rightarrow v_{2}$ and $y_{m} \rightarrow v_{3}$ be three pairwise non-incident arcs from $D_{p}$ to $V(C)$. Let $v_{i}^{\prime} \in D_{i}$ $(2 \leq i \leq p-1)$. If one of the vertices $x_{s}, x_{t}$ or $x_{m}$ does not belong to any GodOelcycle $C_{1}$ of $D_{1}$, say $x_{s} \notin C_{1}$, then $C_{1}$ and $\left(v_{1} x_{s} v_{2}^{\prime} v_{3}^{\prime} \cdots v_{p-1}^{\prime} y_{t}^{+} y_{t}^{++} \cdots y_{t} v_{2} v_{3} v_{1}\right.$ or $\left.v_{1} x_{s} v_{2}^{\prime} v_{3}^{\prime} \cdots v_{p-1}^{\prime} y_{t}^{++} \cdots y_{t} v_{2} v_{3} v_{1}\right)$ are two weakly complementary cycles, a contradiction. Hence we have $\left\{x_{s}, x_{t}, x_{m}\right\} \subseteq V\left(C_{1}\right)$. Analogously, we observe that $\left\{y_{s}, y_{t}, y_{m}\right\} \subseteq V\left(C_{p}\right)$ for a GodOel-cycle $C_{p}$ of $D\left[D_{p}\right]$.

Subcase 1.2.1. Assume that $p=2$.

Subcase 1.2.1.1. Assume that $x_{t}$ is on the oriented path along $C_{1}$ from $x_{m}$ to $x_{s}$ and that $y_{m}$ is on the path along $C_{2}$ from $y_{s}$ to $y_{t}$. Then we have one of the cycles $v_{1} x_{s} x_{s}^{+} \cdots x_{t}^{-} y_{t}^{+} y_{t}^{++} \cdots y_{m} v_{3} v_{1}$ and $v_{1} x_{s} x_{s}^{+} \cdots x_{t}^{--} y_{t}^{+} y_{t}^{++} \cdots y_{m} v_{3} v_{1}$. Let this cycle be called $C_{1}^{\prime}$. If we are not in the case that $x_{t}^{+}=x_{s}, y_{m}^{+}=y_{t}$ and $V\left(x_{t}\right)=V\left(y_{t}\right)$, then there are the cycles $v_{2} x_{t} x_{t}^{+} \cdots x_{s}^{-} y_{m}^{+} y_{m}^{++} \cdots y_{t} v_{2}$ or $v_{2} x_{t} x_{t}^{+} \cdots x_{s}^{--} y_{m}^{+} y_{m}^{++} \cdots y_{t} v_{2}$ or $v_{2} x_{t} x_{t}^{+} \cdots x_{s}^{-} y_{m}^{++} \cdots y_{t} v_{2}$ and $C_{1}^{\prime}$, which contain vertices from all partite sets of $D$, a contradiction.

Hence, let $x_{t}^{+}=x_{s}, y_{m}^{+}=y_{t}$ and $V\left(x_{t}\right)=V\left(y_{t}\right)$. Now we have one of 
the cycles $v_{1} v_{2} x_{t} x_{t}^{+} \cdots x_{m}^{-} y_{t} y_{t}^{+} \cdots y_{s} v_{1}$ and $v_{1} v_{2} x_{t} x_{t}^{+} \cdots x_{m}^{--} y_{t} y_{t}^{+} \cdots y_{s} v_{1}$. Let this cycle be called $C_{1}^{\prime \prime}$. If we are not in the case that $x_{m}^{+}=x_{t}, y_{m}^{-}=y_{s}$ and $V\left(x_{m}\right)=V\left(y_{m}\right)$, then $D$ contains one of the cycles $v_{3} x_{m} x_{m}^{+} \cdots x_{t}^{-} y_{s}^{+} y_{s}^{++} \cdots y_{m} v_{3}$, $v_{3} x_{m} x_{m}^{+} \cdots x_{t}^{--} y_{s}^{+} y_{s}^{++} \cdots y_{m} v_{3}$ and $v_{3} x_{m} x_{m}^{+} \cdots x_{t}^{-} y_{s}^{++} \cdots y_{m} v_{3}$. This cycle and $C_{1}^{\prime \prime}$ are weakly complementary cycles, a contradiction.

Hence, let $x_{m}^{+}=x_{t}, y_{m}^{-}=y_{s}$ and $V\left(x_{m}\right)=V\left(y_{m}\right)$. Now we have the cycle $C_{1}^{\prime \prime \prime}=v_{2} v_{3} x_{m} x_{t} v_{2}$. If we are not in the case that $x_{s}^{+}=x_{m}, y_{t}^{+}=y_{s}$ and $V\left(x_{s}\right)=V\left(y_{s}\right)$, then $D$ contains one of the cycles $v_{1} x_{s} x_{s}^{+} \cdots x_{m}^{-} y_{t}^{+} y_{t}^{++} \cdots y_{s} v_{1}$, $v_{1} x_{s} x_{s}^{+} \cdots x_{m}^{--} y_{t}^{+} y_{t}^{++} \cdots y_{s} v_{1}$ and $v_{1} x_{s} x_{s}^{+} \cdots x_{m}^{-} y_{t}^{++} \cdots y_{s} v_{1}$. This cycle and $C_{1}^{\prime \prime \prime}$ are weakly complementary cycles a contradiction.

Hence, let $x_{s}^{+}=x_{m}, y_{t}^{+}=y_{s}$ and $V\left(x_{s}\right)=V\left(y_{s}\right)$. But this implies that $\left|V\left(C_{1}\right)\right|=\left|V\left(C_{2}\right)\right|=3$ and $C_{1}$ and $C_{2}$ consist of vertices from the same partite sets. Consequently we arrive at the weakly complementary cycles $C_{1}$ and $C$, also a contradiction.

Subcase 1.2.1.2. Assume that $x_{t}$ is on the path along $C_{1}$ from $x_{s}$ to $x_{m}$ and that $y_{m}$ is on the path along $C_{2}$ from $y_{s}$ to $y_{t}$. Then there is one of the cycles $v_{2} x_{t} x_{t}^{+} \cdots x_{s}^{-} y_{m}^{+} y_{m}^{++} \cdots y_{t} v_{2}$ and $v_{2} x_{t} x_{t}^{+} \cdots x_{s}^{--} y_{m}^{+} y_{m}^{++} \cdots y_{t} v_{2}$, say this cycle is $C_{1}^{\prime}$. Furthermore, there is one of the cycles $v_{3} v_{1} x_{s} x_{s}^{+} \cdots x_{t}^{-} y_{t}^{+} y_{t}^{++} \cdots y_{m} v_{3}$ and $v_{3} v_{1} x_{s} x_{s}^{+} \cdots x_{t}^{-} y_{t}^{++} \cdots y_{m} v_{3}$. This cycle and $C_{1}^{\prime}$ are weakly complementary cycles, a contradiction.

Subcase 1.2.1.3. Assume that $x_{t}$ is on the path along $C_{1}$ from $x_{s}$ to $x_{m}$ and that $y_{m}$ is on the path along $C_{2}$ from $y_{t}$ to $y_{s}$. Then there is one of the cycles $v_{2} x_{t} x_{t}^{+} \cdots x_{s}^{-} y_{m}^{+} y_{m}^{++} \cdots y_{t} v_{2}$ and $v_{2} x_{t} x_{t}^{+} \cdots x_{s}^{-} y_{m}^{++} \cdots y_{t} v_{2}$. Say this cycle is $C_{1}^{\prime}$. If we are not in the case that $x_{s}^{+}=x_{t}, y_{t}^{+}=y_{m}$ and $V\left(x_{s}\right)=V\left(y_{m}\right)$, then $D$ has one of the cycles $v_{3} v_{1} x_{s} x_{s}^{+} \cdots x_{t}^{-} y_{t}^{+} y_{t}^{++} \cdots y_{m} v_{3}, v_{3} v_{1} x_{s} x_{s}^{+} \cdots x_{t}^{--} y_{t}^{+} y_{t}^{++} \cdots y_{m} v_{3}$ and $v_{3} v_{1} x_{s} x_{s}^{+} \cdots x_{t}^{-} y_{t}^{++} \cdots y_{m} v_{3}$. This cycle and $C_{1}^{\prime}$ are weakly complementary cycles of $D$, a contradiction.

Hence, let $x_{s}^{+}=x_{t}, y_{t}^{+}=y_{m}$ and $V\left(x_{s}\right)=V\left(y_{m}\right)$. Now we have one of the cycles $v_{1} x_{s} x_{s}^{+} \cdots x_{m}^{-} y_{m} y_{m}^{+} \cdots y_{s} v_{1}$ and $v_{1} x_{s} x_{s}^{+} \cdots x_{m}^{--} y_{m} y_{m}^{+} \cdots y_{s} v_{1}$. Let this cycle be called $C_{1}^{\prime \prime}$. If we are not in the case that $x_{m}^{+}=x_{s}, y_{s}^{+}=y_{t}$ and $V\left(x_{m}\right)=V\left(y_{t}\right)$, then there is one of the cycles $v_{2} v_{3} x_{m} x_{m}^{+} \cdots x_{s}^{-} y_{s}^{+} y_{s}^{++} \cdots y_{t} v_{2}$, $v_{2} v_{3} x_{m} x_{m}^{+} \cdots x_{s}^{--} y_{s}^{+} y_{s}^{++} \cdots y_{t} v_{2}$ and $v_{2} v_{3} x_{m} x_{m}^{+} \cdots x_{s}^{-} y_{s}^{++} \cdots y_{t} v_{2}$. This cycle and $C_{1}^{\prime \prime}$ are weakly complementary cycles, a contradiction.

Hence, let $x_{m}^{+}=x_{s}, y_{s}^{+}=y_{t}$ and $V\left(x_{m}\right)=V\left(y_{t}\right)$. Then $D$ contains the cycle $C_{1}^{\prime \prime \prime}=v_{3} x_{m} y_{m} v_{3}$. If we are not in the case that $x_{t}^{+}=x_{m}, y_{m}^{+}=y_{s}$ and $V\left(x_{t}\right)=V\left(y_{s}\right)$, then there is one of the cycles $v_{1} v_{2} x_{t} x_{t}^{+} \cdots x_{m}^{-} y_{m}^{+} y_{m}^{++} \cdots y_{s} v_{1}$, $v_{1} v_{2} x_{t} x_{t}^{+} \cdots x_{m}^{--} y_{m}^{+} y_{m}^{++} \cdots y_{s} v_{1}$ and $v_{1} v_{2} x_{t} x_{t}^{+} \cdots x_{m}^{-} y_{m}^{++} \cdots y_{s} v_{1}$. This cycle and $C_{1}^{\prime \prime \prime}$ are weakly cycle complementary.

Hence, let $x_{t}^{+}=x_{m}, y_{m}^{+}=y_{s}$ and $V\left(x_{t}\right)=V\left(y_{s}\right)$. This is possible only if $\left|V\left(C_{1}\right)\right|=\left|V\left(C_{2}\right)\right|=3$ and both cycles contain vertices from the same partite sets. Consequently, we deduce that $C$ and $C_{1}$ are weakly complementary cycles, a contradiction. 
Subcase 1.2.1.4. Assume that $x_{t}$ is on the path along $C_{1}$ from $x_{m}$ to $x_{s}$ and that $y_{m}$ is on the path along $C_{2}$ from $y_{t}$ to $y_{s}$. Then $D$ contains $v_{1} v_{2} x_{t} x_{t}^{+} \cdots x_{m}^{-} y_{m}^{+} y_{m}^{++} \cdots y_{s} v_{1}$ or $v_{1} v_{2} x_{t} x_{t}^{+} \cdots x_{m}^{--} y_{m}^{+} y_{m}^{++} \cdots y_{s} v_{1}$, say this cycle is $C_{1}^{\prime}$. Furthermore, there is one of the cycles $v_{3} x_{m} x_{m}^{+} \cdots x_{t}^{-} y_{s}^{+} y_{s}^{++} \cdots y_{m} v_{3}$ and $v_{3} x_{m} x_{m}^{+} \cdots x_{t}^{-} y_{s}^{++} \cdots y_{m} v_{3}$. This cycle and $C_{1}^{\prime}$ are weakly complementary cycles, a contradiction.

Subcase 1.2.2. Assume that $p \geq 3$. If all partite sets appearing in $D_{2} \cup D_{3} \cup \cdots \cup D_{p-1}$ also appear in $D_{1}$ or $D_{p}$ or $V(C)$, then we find the same weakly complementary cycles as in Subcase 1.2.1. If there are vertices of new partite sets in $D_{2} \cup D_{3} \cup$ $\cdots \cup D_{p-1}$, then it is easy to see that these vertices can be inserted into the cycles of Subcase 1.2.1.

Case 2. Assume that there is exactly one vertex set $D_{i}(1 \leq i \leq p)$, which induces a non-trivial strong component of $D-V(C)$.

Subcase 2.1. Assume that $1<i<p$. Let $C_{i}$ be an arbitrary GodOel-cycle of $D_{i}$ and let $v_{j}^{\prime} \in D_{j}$ for all $j \in\{1,2, \cdots, p\} \backslash\{i\}$. If $p \geq 4$ or $V\left(v_{1}^{\prime}\right) \neq$ $V\left(v_{p}^{\prime}\right)$, then $D$ contains one of the cycles $v_{1}^{\prime} v_{2}^{\prime} \cdots v_{i-1}^{\prime} v_{i+1}^{\prime} v_{i+2}^{\prime} \cdots v_{p}^{\prime} v_{1} v_{2} v_{3} v_{1}^{\prime}$, $v_{1}^{\prime} v_{2}^{\prime} \cdots v_{i-2}^{\prime} v_{i+1}^{\prime} v_{i+2}^{\prime} \cdots v_{p}^{\prime} v_{1} v_{2} v_{3} v_{1}^{\prime}$ and $v_{1}^{\prime} v_{2}^{\prime} \cdots v_{i-1}^{\prime} v_{i+2}^{\prime} v_{i+3}^{\prime} \cdots v_{p}^{\prime} v_{1} v_{2} v_{3} v_{1}^{\prime}$. This cycle and $C_{i}$ are weakly complementary cycles, a contradiction. Hence, let $p=3$ and $V\left(v_{1}^{\prime}\right)=V\left(v_{3}^{\prime}\right)$.

Suppose that $\left|D_{1}\right|,\left|D_{3}\right| \geq 2$ with $\left\{v_{1}^{\prime}, v_{1}^{\prime \prime}\right\} \subseteq D_{1}$ and $\left\{v_{3}^{\prime}, v_{3}^{\prime \prime}\right\} \subseteq D_{3}$. Let $x_{i} \in V\left(C_{i}\right)$ be arbitrary such that $x_{i} \notin V\left(v_{1}^{\prime}\right)$. Then $D$ contains the cycle $C_{1}^{\prime}=v_{1}^{\prime} x_{i} v_{3}^{\prime} v_{1} v_{1}^{\prime}$. Furthermore there is one of the cycles $v_{1}^{\prime \prime} x_{i}^{+} x_{i}^{++} \cdots x_{i}^{-} v_{3}^{\prime \prime} v_{2} v_{3} v_{1}^{\prime \prime}$, $v_{1}^{\prime \prime} x_{i}^{++} \cdots x_{i}^{-} v_{3}^{\prime \prime} v_{2} v_{3} v_{1}^{\prime \prime}, v_{1}^{\prime \prime} x_{i}^{+} x_{i}^{++} \cdots x_{i}^{--} v_{3}^{\prime \prime} v_{2} v_{3} v_{1}^{\prime \prime}$ and $v_{1}^{\prime \prime} x_{i}^{++} \cdots x_{i}^{--} v_{3}^{\prime \prime} v_{2} v_{3} v_{1}^{\prime \prime}$. This cycle and $C_{1}^{\prime}$ are weakly complementary in $D$, a contradiction.

It follows that $\left|D_{1}\right|=1$ or $\left|D_{3}\right|=1$. Without loss of generality, let $D_{3}=\left\{v_{3}^{\prime}\right\}$. Suppose that $\left|D_{1}\right| \geq 2$ and $\left\{v_{1}^{\prime}, v_{1}^{\prime \prime}\right\} \subseteq D_{1}$. Because of $\kappa(D) \geq 3$ we conclude that there are vertices $x_{j} \in D_{2}$ and $v_{m} \in V(C)$ such that $x_{j} \rightarrow v_{m}$, say $x_{j} \rightarrow v_{1}$. Let $C_{2}=C_{2}\left(x_{j}\right)$. If $x_{j} \notin V\left(v_{1}^{\prime}\right)$, then there is the cycle $C_{1}^{\prime}=v_{1}^{\prime} x_{j} v_{1} v_{1}^{\prime}$. Furthermore $D$ contains one of the cycles $v_{1}^{\prime \prime} x_{j}^{+} x_{j}^{++} \cdots x_{j}^{-} v_{3}^{\prime} v_{2} v_{3} v_{1}^{\prime \prime}, v_{1}^{\prime \prime} x_{j}^{++} \cdots x_{j}^{-} v_{3}^{\prime} v_{2} v_{3} v_{1}^{\prime \prime}$, $v_{1}^{\prime \prime} x_{j}^{+} x_{j}^{++} \cdots x_{j}^{--} v_{3}^{\prime} v_{2} v_{3} v_{1}^{\prime \prime}$ and $v_{1}^{\prime \prime} x_{j}^{++} \cdots x_{j}^{--} v_{3}^{\prime} v_{2} v_{3} v_{1}^{\prime \prime}$. This cycle and $C_{1}^{\prime}$ are weakly complementary cycles, a contradiction. If $x_{j} \in V\left(v_{1}^{\prime}\right)$, then $D$ contains the weakly complementary cycles $C$ and $C_{2}$, also a contradiction.

Hence, it remains to treat the case that $D_{1}=\left\{v_{1}^{\prime}\right\}$ and $D_{3}=\left\{v_{3}^{\prime}\right\}$. Let $C_{2}$ be a GodOel-cycle of $D\left[D_{2}\right]$. If there is a vertex $v_{2}^{\prime} \in D_{2}$ such that $v_{1}^{\prime} \in V\left(v_{2}^{\prime}\right)$, then $C$ and $C_{2}$ are two weakly complementary cycles a contradiction. Consequently, let $v_{1}^{\prime} \notin V\left(v_{2}^{\prime}\right)$ for all $v_{2}^{\prime} \in D_{2}$. Suppose that there is a vertex $v_{2}^{\prime} \in D_{2}-V\left(C_{2}\right)$. Then $D$ contains the weakly complementary cycles $C_{2}$ and $v_{1} v_{1}^{\prime} v_{2}^{\prime} v_{3}^{\prime} v_{2} v_{3} v_{1}$, also a contradiction. Hence, let $D_{2}=V\left(C_{2}\right)$. Since $\kappa(D)=3$ there are vertices $x_{j} \in D_{2}$ and $v_{m} \in V(C)$ such that $x_{j} \rightarrow v_{m}$. If there is a vertex $x_{p} \in D_{2}$ with $x_{p} \neq x_{j}$ and $x_{p} \neq x_{j}^{+}$such that $v_{m}^{+} \rightarrow x_{p}$ or $v_{m} \rightarrow x_{p}$, then $D$ contains the weakly complementary cycles $x_{p} x_{p}^{+} \cdots x_{j} v_{m} v_{m}^{+} x_{j}$ and $v_{1}^{\prime} x_{j}^{+} x_{j}^{++} \cdots x_{p}^{-} v_{3}^{\prime} v_{m}^{-} v_{1}^{\prime}$ or $x_{p} x_{p}^{+} \cdots x_{j} v_{m} x_{p}$ and $v_{1}^{\prime} x_{j}^{+} x_{j}^{++} \cdots x_{p}^{-} v_{3}^{\prime} v_{m}^{+} v_{m}^{-} v_{1}^{\prime}$, in both cases a contradiction. If $v_{m}^{+} \rightarrow x_{j}$, then we 
find the two weakly complementary cycles $x_{j} v_{m} v_{m}^{+} x_{j}$ and $v_{1}^{\prime} x_{j}^{+} x_{j}^{++} \cdots x_{j}^{-} v_{3}^{\prime} v_{m}^{+} v_{m}^{-} v_{1}^{\prime}$, also a contradiction. Altogether, we see that $\left(D_{2}-\left\{x_{j}^{+}\right\}\right) \sim\left\{v_{m}, v_{m}^{+}\right\}$. Since every vertex of $V(C)$ has an outer neighbor in $D_{2}$ we conclude that $\left\{v_{m}, v_{m}^{+}\right\} \rightarrow x_{j}^{+}$. Moreover, we obviously have $x_{j} \rightarrow v_{m}^{+}$or $x_{j}^{-} \rightarrow v_{m}^{+}$and $x_{j} \in V\left(v_{m}^{+}\right)$.

First, let $x_{j} \rightarrow v_{m}^{+}$. Analogously as above and noticing that $v_{m}^{++}=v_{m}^{-}$we deduce that in this case $\left(D_{2}-\left\{x_{j}^{+}\right\}\right) \leadsto v_{m}^{-} \rightarrow x_{j}^{+}$, and thus

$$
\left\{v_{m}, v_{m}^{+}, v_{m}^{-}\right\} \rightarrow x_{j}^{+} \rightarrow x_{j}^{++} \leadsto\left\{v_{m}, v_{m}^{+}, v_{m}^{-}\right\} .
$$

Now $D-\left\{v_{1}^{\prime}, x_{j}^{+}\right\}$is not strong, a contradiction to $\kappa(D)=3$.

Second, let $x_{j}^{-} \rightarrow v_{m}^{+}$and $x_{j} \in V\left(v_{m}^{+}\right)$. Analogously as above and noticing that $v_{m}^{++}=v_{m}^{-}$we conclude that $\left(D_{2}-\left\{x_{j}\right\}\right) \sim v_{m}^{-} \rightarrow x_{j}$, and thus $D$ contains the weakly complementary cycles $x_{j}^{-} v_{m}^{+} x_{j}^{+} x_{j}^{++} \cdots x_{j}^{-}$and $v_{1}^{\prime} x_{j} v_{3}^{\prime} v_{m}^{-} v_{m} v_{1}^{\prime}$, a contradiction.

Subcase 2.2. Assume that $i=1$. If there is an arc leading from $V(C)$ to $D_{2}$, say $v_{3} \rightarrow v_{2}^{\prime}$, then there are the weakly complementary cycles $v_{1} v_{2} v_{3} v_{2}^{\prime} v_{3}^{\prime} \cdots v_{p}^{\prime} v_{1}$ and the GodOel-cycle of $D_{1}$, a contradiction. Hence, let $D_{2} \leadsto V(C)$. If there are vertices $v_{m} \in V(C)$ and $v_{1}^{\prime} \in D_{1}$ such that $v_{m} \rightarrow v_{1}^{\prime}$ and $v_{1}^{\prime}$ is not contained in a GodOel-cycle $C_{1}$ of $D\left[D_{1}\right]$, then $C_{1}$ and $v_{m} v_{1}^{\prime} v_{2}^{\prime} \cdots v_{p}^{\prime} v_{m}^{+} v_{m}^{-} v_{m}$ are two weakly complementary cycles of $D$, also a contradiction. Consequently, let all vertices $v_{1}^{\prime} \in D_{1}$ that are outer neighbors of a vertex of $V(C)$ be on every GodOel-cycle of $D\left[D_{1}\right]$.

Subcase 2.2.1. Assume that $p \geq 3$. Furthermore, let $C^{\prime}=x_{1} x_{2} \cdots x_{l} x_{1}$ be a GodOel-cycle of $D\left[D_{1}\right]$. Since $\kappa(D)=3$ there are three pairwise non-incident arcs leading from $V(C)$ to $D_{1}$, say $v_{1} \rightarrow x_{s}, v_{2} \rightarrow x_{t}$ and $v_{3} \rightarrow x_{u}$.

Subcase 2.2.1.1. Assume that $x_{u}$ is on the path along $C^{\prime}$ from $x_{s}$ to $x_{t}$. If $v_{3} \notin V\left(v_{2}^{\prime}\right)$, then there is one of the cycles $v_{1} x_{s} x_{s}^{+} \cdots x_{t}^{-} v_{2}^{\prime} v_{3} v_{1}$ and $v_{1} x_{s} x_{s}^{+} \cdots x_{t}^{--} v_{2}^{\prime} v_{3} v_{1}$, and if $v_{3} \in V\left(v_{2}^{\prime}\right)$, then $D$ contains one of the cycles $v_{1} x_{s} x_{s}^{+} \cdots x_{t}^{-} v_{2}^{\prime} v_{1}$ and $v_{1} x_{s} x_{s}^{+} \cdots x_{t}^{--} v_{2}^{\prime} v_{1}$. In any case, let this cycle be called $C^{\prime \prime}$. If $x_{s}^{-} \notin V\left(v_{p}^{\prime}\right)$ or if $x_{s}^{-} \in$ $V\left(v_{p}^{\prime}\right)$ and $p \geq 4$, then $C^{\prime \prime}$ and $v_{2} x_{t} x_{t}^{+} \cdots x_{s}^{-} v_{3}^{\prime} v_{4}^{\prime} \cdots v_{p}^{\prime} v_{2}$ or $v_{2} x_{t} x_{t}^{+} \cdots x_{s}^{-} v_{4}^{\prime} \cdots v_{p}^{\prime} v_{2}$ are two weakly complementary cycles, a contradiction. If $p=3, x_{s}^{-} \in V\left(v_{3}^{\prime}\right)$ and $x_{s}^{-} \neq x_{t}$, then $C^{\prime \prime}$ and $v_{2} x_{t} x_{t}^{+} \cdots x_{s}^{--} v_{3}^{\prime} v_{2}$ are weakly complementary cycles of $D$, also a contradiction. Consequently, let $p=3, x_{s}^{-} \in V\left(v_{3}^{\prime}\right)$ and $x_{s}^{-}=x_{t}$. If $v_{2} \in V\left(v_{2}^{\prime}\right)$, then $C$ and $C^{\prime}$ are two weakly complementary cycles of $D$, a contradiction. Hence, let $v_{2} \notin V\left(v_{2}^{\prime}\right)$. But now we arrive at the weakly complementary cycles $v_{1} x_{s} x_{s}^{+} \cdots x_{t}^{-} v_{3}^{\prime} v_{3} v_{1}$ and $v_{2} x_{t} v_{2}^{\prime} v_{2}$, a contradiction.

Subcase 2.2.1.2. Assume that $x_{u}$ is on the path along $C^{\prime}$ from $x_{t}$ to $x_{s}$.

First, let $v_{2}^{\prime} \in V\left(v_{2}\right)$. Then $D$ contains one of the cycles $v_{3} x_{u} x_{u}^{+} \cdots x_{t}^{-} v_{2}^{\prime} v_{3}$ and $v_{3} x_{u} x_{u}^{+} \cdots x_{t}^{--} v_{2}^{\prime} v_{3}$. Let this cycle be called $C^{\prime \prime}$. If $p \geq 4$ or if $p=3$ and $x_{u}^{-} \notin V\left(v_{3}^{\prime}\right)$, then $C^{\prime \prime}$ and $v_{2} x_{t} x_{t}^{+} \cdots x_{u}^{-} v_{3}^{\prime} v_{4}^{\prime} \cdots v_{p}^{\prime} v_{1} v_{2}$ or $v_{2} x_{t} x_{t}^{+} \cdots x_{u}^{-} v_{4}^{\prime} \cdots v_{p}^{\prime} v_{1} v_{2}$ are two weakly complementary cycles, a contradiction. In the remaining case that $p=3$ and $x_{u}^{-} \in V\left(v_{3}^{\prime}\right)$ the GodOel-cycle in $D\left[D_{1}\right]$ and the cycle $C$ are two weakly complementary cycles of $D$, also a contradiction. 
Second, let $v_{2}^{\prime} \notin V\left(v_{2}\right)$. Then $D$ contains one of the cycles $v_{2} x_{t} x_{t}^{+} \cdots x_{s}^{-} v_{2}^{\prime} v_{2}$ and $v_{2} x_{t} x_{t}^{+} \cdots x_{s}^{--} v_{2}^{\prime} v_{2}$. Let this cycle be called $\tilde{C}$. If $x_{t}^{-} \notin V\left(v_{p}^{\prime}\right)$ or if $x_{t}^{-} \in V\left(v_{p}^{\prime}\right)$ and $p \geq 4$, then $\tilde{C}$ and $v_{1} x_{s} x_{s}^{+} \cdots x_{t}^{-} v_{3}^{\prime} v_{4}^{\prime} \cdots v_{p}^{\prime} v_{3} v_{1}$ or $v_{1} x_{s} x_{s}^{+} \cdots x_{t}^{-} v_{4}^{\prime} \cdots v_{p}^{\prime} v_{3} v_{1}$ are two weakly complementary cycles, a contradiction. If $p=3, x_{t}^{-} \in V\left(v_{3}^{\prime}\right)$ and $x_{t}^{-} \neq x_{s}$, then $\tilde{C}$ and $v_{1} x_{s} x_{s}^{+} \cdots x_{t}^{--} v_{3}^{\prime} v_{3} v_{1}$ are weakly complementary cycles, also a contradiction. Hence, let $p=3, x_{t}^{-} \in V\left(v_{3}^{\prime}\right)$ and $x_{t}^{-}=x_{s}$. If $v_{3} \in V\left(v_{2}^{\prime}\right)$ or $x_{s} \in V\left(v_{2}^{\prime}\right)$, then $C$ and $C^{\prime}$ are two weakly complementary cycles, a contradiction. Otherwise if $v_{3}, x_{s} \notin V\left(v_{2}^{\prime}\right)$, then $v_{2} x_{t} x_{t}^{+} \cdots x_{s}^{-} v_{3}^{\prime} v_{2}$ and $v_{1} x_{s} v_{2}^{\prime} v_{3} v_{1}$ are weakly complementary cycles, again a contradiction.

Subcase 2.2.2. Assume that $p=2$. If there are vertices $v_{2}^{\prime} \in D_{2}$ and $v_{1}^{\prime} \in D_{1}$ such that $v_{2}^{\prime} \in V\left(v_{1}^{\prime}\right)$, then $C$ and the GodOel-cycle of $D\left[D_{1}\right]$ are weakly complementary cycles, a contradiction. Consequently, we have $D_{1} \rightarrow D_{2} \rightarrow V(C)$. Let $C^{\prime}=x_{1} x_{2} \cdots x_{l} x_{1}$ be the GodOel-cycle of $D\left[D_{1}\right]$. If there are vertices $v_{i} \in V(C)$ and $v_{1}^{\prime} \in\left(D_{1}-V\left(C^{\prime}\right)\right)$ such that $v_{i} \rightarrow v_{1}^{\prime}$, then $C^{\prime}$ and $v_{i} v_{1}^{\prime} v_{2}^{\prime} v_{i}^{+} v_{i}^{-} v_{i}$ are weakly complementary cycles, a contradiction. Consequently, let $\left(D_{1}-V\left(C^{\prime}\right)\right) \leadsto V(C)$. Because of $\kappa(D)=3$ we observe that there are three pairwise non-incident arcs leading from $V(C)$ to $V\left(C^{\prime}\right)$, say $v_{1} \rightarrow x_{s}, v_{2} \rightarrow x_{t}$ and $v_{3} \rightarrow x_{u}$. If $\left|D_{2}\right| \geq 2$ with $\left\{v_{2}^{\prime}, v_{2}^{\prime \prime}\right\} \subseteq D_{2}$, then $D$ contains the weakly complementary cycles $v_{1} x_{s} x_{s}^{+} \cdots x_{t}^{-} v_{2}^{\prime} v_{3} v_{1}$ and $v_{2} x_{t} x_{t}^{+} \cdots x_{s}^{-} v_{2}^{\prime \prime} v_{2}$, a contradiction. Hence, let $\left|D_{2}\right|=1$.

Suppose that there are vertices $v_{i}, x_{j}$ and $x_{s}$ with $i \in\{1,2,3\}, j \in\{1,2, \cdots, l\}$ and $s \in\{1,2, \cdots, l\} \backslash\{j-2, j-1, j\}$ such that $v_{i} \rightarrow x_{j}$ and $x_{j-1} \rightarrow$ $x_{s}$. Then $D$ contains the weakly complementary cycles $x_{j-1} x_{s} x_{s+1} \cdots x_{j-1}$ and $v_{i} x_{j} x_{j+1} \cdots x_{s-1} v_{2}^{\prime} v_{i}^{+} v_{i}^{-} v_{i}$, a contradiction. This leads to

Claim 1. If $l \geq 4$ and $x_{j} \in V\left(C^{\prime}\right)$ is the outer neighbor of a vertex of $V(C)$, then it follows that $x_{s} \leadsto x_{j-1}$ for all $s \in\{1,2, \cdots, l\} \backslash\{j-2, j-1, j\}$.

Claim 1 immediately implies the following claim.

Claim 2. If $l \geq 4$ and there are vertices $v_{i}, v_{m} \in V(C)$ and $x_{j}, x_{p} \in V\left(C^{\prime}\right)$ with $j \notin\{p+1, p, p-1\}$ such that $v_{i} \rightarrow x_{j}$ and $v_{m} \rightarrow x_{p}$, then it follows that $V\left(x_{j-1}\right)=V\left(x_{p-1}\right)$.

Let $\left\{x_{s}, x_{t}, x_{u}\right\}=\left\{x_{p_{1}}, x_{p_{2}}, x_{p_{3}}\right\}$. If we pass the vertices of $V\left(C^{\prime}\right)$ along its orientation, then, without loss of generality, let this three vertices be appear in the order $x_{p_{1}}, x_{p_{2}}, x_{p_{3}}$ such that $p_{1}-p_{3} \geq \max \left\{p_{3}-p_{2}, p_{2}-p_{1}\right\}$ (all indices taken modulo $l$ ). Furthermore, let $\left\{v_{i}, v_{j}, v_{m}\right\}=\left\{v_{1}, v_{2}, v_{3}\right\}$ such that $v_{i} \rightarrow x_{p_{1}}, v_{j} \rightarrow x_{p_{2}}$ and $v_{m} \rightarrow x_{p_{3}}$. If $p_{1} \geq p_{3}+3$ (modulo $l$ ), then Claim 2 implies that $V\left(x_{p_{3}-1}\right)=$ $V\left(x_{p_{1}-1}\right)$ and with Claim 1 we conclude that $x_{p_{1}-2} \rightarrow x_{p_{3}-1}$. Now we observe that $D$ contains the weakly complementary cycles $x_{p_{1}-2} x_{p_{3}-1} x_{p_{3}} x_{p_{3}+1} \cdots x_{p_{1}-2}$ and $v_{i} x_{p_{1}} x_{p_{1}+1} \cdots x_{p_{3}-2} v_{2}^{\prime} v_{i}^{+} v_{i}^{-} v_{i}$, a contradiction. Hence, let $p_{1} \leq p_{3}+2$ (modulo $l$ ). This immediately yields that $l \leq 6$.

Subcase 2.2.2.1. Assume that $l=6$. In this case $p_{1} \leq p_{3}+2$ (modulo $l$ ) implies that, without loss of generality, $p_{1}=1, p_{2}=3$ and $p_{3}=5$. Applying Claim 2, we see that $V\left(x_{2}\right)=V\left(x_{4}\right)=V\left(x_{6}\right)$. Furthermore, with Claim 1 it follows that $x_{1} \rightarrow x_{4}$. 
Now it is obvious that $D$ contains the weakly complementary cycles $x_{1} x_{4} x_{5} x_{6} x_{1}$ and $v_{j} x_{3} v_{2}^{\prime} v_{j}^{+} v_{j}^{-} v_{j}$, a contradiction.

Subcase 2.2.2.2. Assume that $l=5$. Then the fact that $p_{1} \leq p_{3}+2$ (modulo $l$ ) implies that, without loss of generality, $p_{1}=1, p_{2}=3$ and $p_{3}=4$ or $p_{1}=1, p_{2}=2$ and $p_{3}=4$. If $p_{1}=1, p_{2}=3$ and $p_{3}=4$, then Claim 2 implies that $V\left(x_{3}\right)=V\left(x_{5}\right)$ and $V\left(x_{2}\right)=V\left(x_{5}\right)$, a contradiction. And if $p_{1}=1, p_{2}=2$ and $p_{3}=4$, then Claim 2 yields $V\left(x_{3}\right)=V\left(x_{1}\right)$ and $V\left(x_{3}\right)=V\left(x_{5}\right)$, also a contradiction.

Subcase 2.2.2.3. Assume that $l=4$. Without loss of generality, we may suppose that $p_{1}=1, p_{2}=2$ and $p_{3}=3$. Claim 2 yields $V\left(x_{2}\right)=V\left(x_{4}\right)$ and with Claim 1 we conclude that $x_{3} \rightarrow x_{1}$ or $x_{3} \in V\left(x_{1}\right)$.

First, let $x_{3} \rightarrow x_{1}$. Then we conclude that $x_{4} \leadsto V(C)$ since otherwise if there is a vertex $v_{l} \in V(C)$ such that $v_{l} \rightarrow x_{4}$, then $D$ contains the weakly complementary cycles $v_{l} x_{4} v_{2}^{\prime} v_{l}^{+} v_{l}^{-} v_{l}$ and $x_{1} x_{2} x_{3} x_{1}$, a contradiction. Let $v_{m} \in V(C)$ such that $v_{m} \rightarrow x_{2}$. Then $D$ contains the cycle $C^{\prime \prime}:=v_{m} x_{2} x_{3} x_{4} v_{m}$. If $v_{m}^{-} \rightarrow x_{1}$, then $C^{\prime \prime}$ and $v_{m}^{-} x_{1} v_{2}^{\prime} v_{m}^{+} v_{m}^{-}$are weakly complementary cycles, a contradiction. Hence, let $x_{1} \leadsto v_{m}^{-}$, and thus $v_{m}^{-} \rightarrow x_{3}$ and $v_{m}^{+} \rightarrow x_{1}$. If $x_{4} \rightarrow v_{m}^{-}$, then $x_{4} v_{m}^{-} x_{3} x_{4}$ and $v_{m} v_{m}^{+} x_{1} v_{2}^{\prime} v_{m}$ contain all partite sets of $D$, a contradiction. Consequently, we have $V\left(x_{4}\right)=V\left(v_{m}^{-}\right)$. But now $D$ contains the weakly complementary cycles $C^{\prime \prime}$ and $v_{m}^{+} x_{1} v_{2}^{\prime} v_{m}^{+}$, a contradiction.

Second, let $x_{3} \in V\left(x_{1}\right)$. Suppose that there is a vertex $v_{1}^{\prime} \in\left(D_{1}-V\left(C^{\prime}\right)\right)$. Then $v_{1}^{\prime}$ is on a GodOel-cycle $\tilde{C}$ of $D\left[D_{1}\right]$. If $|V(\tilde{C})| \geq 6$, then with Subcase 2.2.2.1 we arrive at a contradiction. Hence, let $|V(\tilde{C})|=4$. Since $\left(D_{1}-V(\tilde{C})\right) \sim V(C)$ and $\left(D_{1}-V\left(C^{\prime}\right)\right) \leadsto V(C)$, we conclude that $v_{1}^{\prime} \in V\left(x_{4}\right)$ and $V(\tilde{C})=\left(V\left(C^{\prime}\right) \cup\right.$ $\left.\left\{v_{1}^{\prime}\right\}\right) \backslash\left\{x_{4}\right\}$. So in any case, if $D_{1}-V\left(C^{\prime}\right)$ is empty or not, we observe that $d_{D-V(C)}^{+}\left(x_{2}\right)=d_{D-V(C)}^{+}\left(x_{4}\right)=2$, and thus $x_{2}$ as well as $x_{4}$ has an outer neighbor in $V(C)$. Without loss of generality, let $v_{1} \rightarrow x_{1}$.

Assume that $v_{2} \rightarrow x_{3}$ and $v_{3} \rightarrow x_{2}$. If $x_{3} \rightarrow v_{1}$, then the cycles $x_{3} v_{1} v_{2} x_{3}$ and $v_{3} x_{2} v_{2}^{\prime} v_{3}$ contain vertices from all partite sets of $D$, a contradiction. Hence, let $v_{1} \rightarrow x_{3}$. If $x_{4} \rightarrow v_{1}$, then $D$ contains the weakly complementary cycles $x_{4} v_{1} x_{3} x_{4}$ and $v_{2} v_{3} x_{2} v_{2}^{\prime} v_{2}$, also a contradiction. Consequently, we have $v_{1} \leadsto x_{4}$. If $x_{4} \rightarrow v_{3}$ and $v_{1} \notin V\left(x_{4}\right)$, then $x_{4} v_{3} v_{1} x_{4}$ and $v_{2} x_{3} v_{2}^{\prime} v_{2}$ are weakly complementary cycles, a contradiction. If $v_{1} \in V\left(x_{4}\right)$ and $x_{1} \rightarrow v_{2}$, then $x_{1} v_{2} x_{3} x_{4} x_{1}$ and $v_{3} x_{2} v_{2}^{\prime} v_{3}$ are weakly complementary cycles, a contradiction. Hence let $v_{2} \rightarrow x_{1}$ in this case. If $x_{4} \rightarrow v_{3}$ and $v_{1} \in V\left(x_{4}\right)$, then the cycles $x_{4} v_{3} x_{2} x_{3} x_{4}$ and $v_{2} x_{1} v_{2}^{\prime} v_{2}$ contain vertices from all partite sets, also a contradiction. Hence, in all cases we observe that $v_{3} \rightarrow x_{4}$. Consequently, $v_{2}$ is an outer neighbor of $x_{4}$. But now $D$ contains the weakly complementary cycles $x_{4} v_{2} v_{3} x_{4}$ and $v_{1} x_{1} v_{2}^{\prime} v_{1}$, a contradiction.

Assume that $v_{2} \rightarrow x_{2}$ and $v_{3} \rightarrow x_{3}$. If $x_{1} \rightarrow v_{3}$, then $D$ contains the weakly complementary cycles $x_{1} v_{3} v_{1} x_{1}$ and $v_{2} x_{2} v_{2}^{\prime} v_{2}$, a contradiction. Hence, let $v_{3} \rightarrow x_{1}$. If $x_{4} \rightarrow v_{3}$, then $x_{4} v_{3} x_{3} x_{4}$ and $v_{1} v_{2} x_{2} v_{2}^{\prime} v_{1}$ are weakly complementary cycles of $D$, also a contradiction. Consequently, we deduce that $v_{3} \leadsto x_{4}$. If $x_{2} \rightarrow v_{1}$, then $D$ contains the cycles $v_{1} v_{2} x_{2} v_{1}$ and $v_{3} x_{1} v_{2}^{\prime} v_{3}$, a contradiction. Hence, let $v_{1} \sim x_{2}$. If $v_{1} \in V\left(x_{2}\right)$, then $v_{2}$ has to be an outer neighbor of $x_{4}$ and $x_{4} v_{2} x_{2} x_{3} x_{4}$ and 
$v_{3} x_{1} v_{2}^{\prime} v_{3}$ contain vertices from all partite sets of $D$, a contradiction. Hence, let $v_{1} \notin V\left(x_{2}\right)$, and thus $v_{1} \rightarrow x_{2}$. If $x_{4} \rightarrow v_{1}$, then $x_{4} v_{1} x_{2} x_{3} x_{4}$ and $v_{3} x_{1} v_{2}^{\prime} v_{2} v_{3}$ are weakly complementary cycles of $D$, a contradiction. Consequently, let $v_{1} \rightarrow x_{4}$ and $v_{2}$ is an outer neighbor of $x_{4}$. This yields the weakly complementary cycles $x_{4} v_{2} v_{3} x_{3} x_{4}$ and $v_{1} x_{2} v_{2}^{\prime} v_{1}$, a contradiction.

Subcase 2.2.2.4. Assume that $l=3$.

First, let there be a vertex $v_{1}^{\prime} \in\left(D_{1}-V\left(C^{\prime}\right)\right)$. Then $v_{1}^{\prime}$ is on a GodOel-cycle $\tilde{C}$ of $D\left[D_{1}\right]$. If $|V(\tilde{C})| \geq 4$, then the previous subcases yield a contradiction. Hence, let $|V(\tilde{C})|=3$, and thus $x_{i} \in\left(V\left(C^{\prime}\right)-V(\tilde{C})\right)$ for an $1 \leq i \leq 3$. If $v_{j} \rightarrow x_{i}$, then $v_{j} x_{i} v_{2}^{\prime} v_{j}^{+} v_{j}^{-} v_{j}$ and $\tilde{C}$ are weakly complementary cycles, a contradiction.

Second, let $D_{1}=V\left(C^{\prime}\right)$. In this case, $D$ is a tournament of order seven and Theorem 2.2 implies that $D$ is cycle complementary and thus weakly cycle complementary unless $D$ is the tournament $T_{7}$ of Example 2.1. The tournament $T_{7}$ is 3 -connected and not (weakly) cycle complementary.

Subcase 2.3. Assume that $i=p$. Observing the converse $D^{-1}$ of $D$ Subcase 2.2 yields a contradiction.

Case 3. Assume that $D_{i}$ is a set of independent vertices for all $1 \leq i \leq p$. As seen above, it follows that $D_{p} \rightarrow V(C) \rightarrow D_{1}$, and obviously we have $D_{i} \rightarrow D_{i+1}$ for all $1 \leq i \leq p-1$. In the following, if we speak of a vertex $v_{i}^{\prime}$, then we mean that $v_{i}^{\prime} \in \bar{D}_{i}$.

First, we assume that $\left|D_{1}\right| \geq 2$ and $\left|D_{p}\right| \geq 2$ such that $\left\{v_{1}^{\prime}, v_{1}^{\prime \prime}\right\} \subseteq D_{1}$ and $\left\{v_{p}^{\prime}, v_{p}^{\prime \prime}\right\} \subseteq D_{p}$. If the vertices of $D_{1}$ and $D_{p}$ belong to different partite sets, then $D$ contains the weakly complementary cycles $v_{1}^{\prime} v_{p}^{\prime} v_{1} v_{1}^{\prime}$ and $v_{1}^{\prime \prime} v_{2}^{\prime} v_{3}^{\prime} \cdots v_{p-1}^{\prime} v_{p}^{\prime \prime} v_{2} v_{3} v_{1}^{\prime \prime}$, a contradiction. Hence, we have to analyze the case that $v_{1}^{\prime} \in V\left(v_{p}^{\prime}\right)$. The fact that $\kappa(D)=3$ implies that $\left|D_{2} \cup D_{3} \cup \cdots D_{p-1}\right| \geq 3$. If $p=3$, then $v_{1}^{\prime} v_{2}^{\prime} v_{3}^{\prime} v_{1} v_{1}^{\prime}$ and $v_{1}^{\prime \prime} v_{2}^{\prime \prime} v_{3}^{\prime \prime} v_{2} v_{3} v_{1}^{\prime \prime}$ are weakly complementary cycles, if $p=4$, then $v_{1}^{\prime} v_{2}^{\prime} v_{4}^{\prime} v_{1} v_{1}^{\prime}$ and $v_{1}^{\prime \prime} v_{3}^{\prime} v_{4}^{\prime \prime} v_{2} v_{3} v_{1}^{\prime \prime}$ are weakly complementary cycles, and if $p \geq 5$, then $v_{1}^{\prime} v_{2}^{\prime} v_{p}^{\prime} v_{1} v_{1}^{\prime}$ and $v_{1}^{\prime \prime} v_{3}^{\prime} v_{4}^{\prime} \cdots v_{p-1}^{\prime} v_{p}^{\prime \prime} v_{2} v_{3} v_{1}^{\prime \prime}$ or $v_{1}^{\prime \prime} v_{4}^{\prime} v_{5}^{\prime} \cdots v_{p-1}^{\prime} v_{p}^{\prime \prime} v_{2} v_{3} v_{1}$ are weakly complementary cycles, in all cases a contradiction. Hence, it follows that $\left|D_{1}\right|=1$ or $\left|D_{p}\right|=1$. Without loss of generality, let $\left|D_{p}\right|=1$. The fact that $\kappa(D)=3$ yields that $\left|D_{2} \cup D_{3} \cup \cdots \cup D_{p-1}\right| \geq 2$.

Second, we assume that $\left|D_{1}\right| \geq 2$ such that $\left\{v_{1}^{\prime}, v_{1}^{\prime \prime}\right\} \subseteq D_{1}$. The fact that $\kappa(D)=3$ implies that there are vertices $v_{p-1}^{\prime} \in D_{p-1}$ and $v_{j} \in V(C)$, say $j=1$, such that $v_{p-1}^{\prime} \rightarrow v_{1}$. If the vertices of $D_{1}$ and $D_{p}$ belong to different partite sets, then $v_{1}^{\prime} v_{p}^{\prime} v_{3} v_{1}^{\prime}$ and $v_{1}^{\prime \prime} v_{2}^{\prime} v_{3}^{\prime} \cdots v_{p-1}^{\prime} v_{1} v_{2} v_{1}^{\prime \prime}$ contain vertices from all partite sets of $D$, a contradiction. Hence, let $v_{1}^{\prime} \in V\left(v_{p}^{\prime}\right)$. If $p \geq 4$, then $v_{1}^{\prime} v_{2}^{\prime} v_{p}^{\prime} v_{3} v_{1}^{\prime}$ and $v_{1}^{\prime \prime} v_{3}^{\prime} v_{4}^{\prime} \cdots v_{p-1}^{\prime} v_{1} v_{2} v_{1}^{\prime \prime}$ or $v_{1}^{\prime \prime} v_{4}^{\prime} \cdots v_{p-1}^{\prime} v_{1} v_{2} v_{1}^{\prime \prime}$ are weakly complementary cycles of $D$ and if $p=3$, and thus $\left|D_{2}\right| \geq 2$, then $v_{1}^{\prime} v_{2}^{\prime \prime} v_{3}^{\prime} v_{2} v_{3} v_{1}^{\prime}$ and $v_{1}^{\prime \prime} v_{2}^{\prime} v_{1} v_{1}^{\prime \prime}$ are weakly complementary cycles, in all cases a contradiction.

Consequently, it remains to treat the case that $\left|D_{1}\right|=\left|D_{p}\right|=1$. Suppose that $p=3$. Since $\kappa(D)=3$, we conclude that a vertex $v_{2}^{\prime} \in D_{2}$ has at least two outer and two inner neighbors in $V(C)$, a contradiction. Hence, let $p \geq 4$. 
Subcase 3.1. Assume that there are vertices $v_{i} \in V(C), v_{k}^{\prime} \in D_{k}$ and $v_{l}^{\prime} \in D_{l}$ with $2 \leq l \leq k \leq p-1$ such that $v_{k}^{\prime} \rightarrow v_{i} \rightarrow v_{l}^{\prime}$. In this case let $k$ and $l$ be chosen such that $k-l$ is minimal. Obviously, $D$ contains the cycle $C^{\prime}=v_{l}^{\prime} v_{l+1}^{\prime} \cdots v_{k}^{\prime} v_{i} v_{l}^{\prime}$. If $V\left(v_{l-1}^{\prime}\right) \neq V\left(v_{k+1}^{\prime}\right)$, then $C^{\prime}$ and $v_{1}^{\prime} v_{2}^{\prime} \cdots v_{l-1}^{\prime} v_{k+1}^{\prime} v_{k+2}^{\prime} \cdots v_{p}^{\prime} v_{i}^{+} v_{i}^{-} v_{1}^{\prime}$ are weakly complementary cycles, a contradiction. Hence let $V\left(v_{l-1}^{\prime}\right)=V\left(v_{k+1}^{\prime}\right)$. If $l-1 \neq 1$, then $C^{\prime}$ and $v_{1}^{\prime} v_{2}^{\prime} \cdots v_{l-2}^{\prime} v_{k+1}^{\prime} v_{k+2}^{\prime} \cdots v_{p}^{\prime} v_{i}^{+} v_{i}^{-} v_{1}^{\prime}$ are weakly complementary cycles, and if $k+1 \neq p$, then $C^{\prime}$ and $v_{1}^{\prime} v_{2}^{\prime} \cdots v_{l-1}^{\prime} v_{k+2}^{\prime} v_{k+3}^{\prime} \cdots v_{p}^{\prime} v_{i}^{+} v_{i}^{-} v_{1}^{\prime}$ contain vertices from all partite sets of $D$, in both cases a contradiction. Consequently, it remains to treat the case that $l=2, k=p-1$ and $V\left(v_{1}^{\prime}\right)=V\left(v_{p}^{\prime}\right)$.

If $\left|D_{m}\right| \geq 2$ with $m \in\{2, p-1\}$ and $v_{m}^{\prime \prime} \in D_{m}-\left\{v_{m}^{\prime}\right\}$, then $C^{\prime}$ and $v_{1}^{\prime} v_{m}^{\prime \prime} v_{p}^{\prime} v_{i}^{+} v_{i}^{-} v_{1}^{\prime}$ are weakly complementary cycles, a contradiction. Hence let $D_{2}=\left\{v_{2}^{\prime}\right\}$ and $D_{p}=\left\{v_{p}^{\prime}\right\}$. If $p \geq 6$ or $p=5$ and $v_{3}^{\prime} \notin V\left(v_{i}\right)$, then we arrive at a contradiction to the minimality of $k-l$. If $p=5, v_{3}^{\prime} \in V\left(v_{i}\right)$ and $v_{2}^{\prime} \notin V\left(v_{4}^{\prime}\right)$, then $D$ contains the weakly complementary cycles $v_{2}^{\prime} v_{4}^{\prime} v_{i} v_{2}^{\prime}$ and $v_{1}^{\prime} v_{3}^{\prime} v_{5}^{\prime} v_{i}^{+} v_{i}^{-} v_{1}^{\prime}$, a contradiction. If $p=5, v_{3}^{\prime} \in V\left(v_{i}\right)$ and $v_{2}^{\prime} \in V\left(v_{4}^{\prime}\right)$, then the fact that $D-\left\{v_{4}^{\prime}, v_{5}^{\prime}\right\}$ is strongly connected implies that there is an arc leading from $v_{3}^{\prime}$ to $\left\{v_{i}^{+}, v_{i}^{-}\right\}$. If $v_{3}^{\prime} \rightarrow v_{i}^{+}$, then $v_{1}^{\prime} v_{3}^{\prime} v_{i}^{+} v_{i}^{-} v_{1}^{\prime}$ and $v_{5}^{\prime} v_{i} v_{2}^{\prime} v_{5}^{\prime}$ are weakly complementary cycles and if $v_{i}^{+} \rightarrow v_{3}^{\prime} \rightarrow v_{i}^{-}$, then $v_{2}^{\prime} v_{3}^{\prime} v_{i}^{-} v_{i} v_{2}^{\prime}$ and $v_{1}^{\prime} v_{4}^{\prime} v_{5}^{\prime} v_{i}^{+} v_{1}^{\prime}$ contain vertices from all partite sets of $D$, in both cases a contradiction. Finally, if $p=4$, then we arrive at the contradiction that $D-\left\{v_{2}^{\prime}, v_{3}^{\prime}\right\}$ is not strong.

Subcase 3.2. Assume that there are vertices $v_{i} \in V(C), v_{k}^{\prime} \in D_{k}$ and $v_{l}^{\prime} \in D_{l}$ with $2 \leq k<l \leq p-1$ such that $v_{k}^{\prime} \rightarrow v_{i} \rightarrow v_{l}^{\prime}$. In this case let $k$ and $l$ be chosen such that $l-k$ is minimal. If $v_{i}^{+} \rightarrow v_{k}^{\prime}$, then $v_{k}^{\prime} v_{i} v_{i}^{+} v_{k}^{\prime}$ and $v_{1}^{\prime} v_{2}^{\prime} \cdots v_{k-1}^{\prime} v_{k+1}^{\prime} v_{k+2}^{\prime} \cdots v_{p}^{\prime} v_{i}^{-} v_{1}^{\prime}$ or $v_{1}^{\prime} v_{2}^{\prime} \cdots v_{k-1}^{\prime} v_{k+2}^{\prime} \cdots v_{p}^{\prime} v_{i}^{-} v_{1}^{\prime}$ are two weakly complementary cycles of $D$, a contradiction. Hence, let $v_{k}^{\prime} \leadsto v_{i}^{+}$. Analogously, if $v_{l}^{\prime} \rightarrow v_{i}^{-}$, then we see that $v_{l}^{\prime} v_{i}^{-} v_{i} v_{l}^{\prime}$ and $v_{1}^{\prime} v_{2}^{\prime} \cdots v_{l-1}^{\prime} v_{l+1}^{\prime} v_{l+2}^{\prime} \cdots v_{p}^{\prime} v_{i}^{+} v_{1}^{\prime}$ or $v_{1}^{\prime} v_{2}^{\prime} \cdots v_{l-2}^{\prime} v_{l+1}^{\prime} v_{l+2}^{\prime} \cdots v_{p}^{\prime} v_{i}^{+} v_{1}^{\prime}$ are weakly complementary cycles, also a contradiction. Consequently, let $v_{i}^{-} \leadsto v_{l}^{\prime}$. Since $l-k$ is minimal we conclude that $l-k=1$ or $l-k=2$ and $v_{i} \in V\left(v_{k+1}^{\prime}\right)$.

First let $l-k=2$ and $v_{i} \in V\left(v_{k+1}^{\prime}\right)$. If $v_{k+1}^{\prime} \rightarrow v_{i}^{+}$, then $D$ contains the weakly complementary cycles $v_{1}^{\prime} v_{2}^{\prime} \cdots v_{k+1}^{\prime} v_{i}^{+} v_{i}^{-} v_{1}^{\prime}$ and $v_{l}^{\prime} v_{l+1}^{\prime} \cdots v_{p}^{\prime} v_{i} v_{l}^{\prime}$, a contradiction. Hence, let $v_{i}^{+} \rightarrow v_{k+1}^{\prime}$. Analogously, we observe that $v_{k+1}^{\prime} \rightarrow v_{i}^{-}$. If $v_{i}^{-} \in\left(V\left(v_{2}^{\prime}\right) \cup V\left(v_{3}^{\prime}\right) \cup \cdots \cup V\left(v_{p-1}^{\prime}\right)\right)$, then $v_{1}^{\prime} v_{2}^{\prime} \cdots v_{k}^{\prime} v_{i} v_{1}^{\prime}$ and $v_{k+1}^{\prime} v_{k+2}^{\prime} \cdots v_{p}^{\prime} v_{i}^{+} v_{k+1}^{\prime}$ contain vertices from all partite sets of $D$, a contradiction. Consequently, let $v_{i}^{-} \notin\left(V\left(v_{2}^{\prime}\right) \cup V\left(v_{3}^{\prime}\right) \cup \cdots \cup V\left(v_{p-1}^{\prime}\right)\right)$, and thus $v_{i}^{-} \rightarrow v_{l}^{\prime}$. If $v_{i}^{-} \rightarrow v_{k}^{\prime}$, then Subcase 3.1 yields a contradiction. Hence let $v_{k}^{\prime} \rightarrow v_{i}^{-}$. But now $D$ contains the weakly complementary cycles $v_{1}^{\prime} v_{2}^{\prime} \cdots v_{k}^{\prime} v_{i}^{-} v_{1}^{\prime}$ and $v_{k+1}^{\prime} v_{k+2}^{\prime} \cdots v_{p}^{\prime} v_{i}^{+} v_{k+1}^{\prime}$, a contradiction.

Second let $l=k+1$. If $v_{k}^{\prime} \notin V\left(v_{i}^{+}\right)$and thus, as seen above, $v_{k}^{\prime} \rightarrow v_{i}^{+}$, then $v_{1}^{\prime} v_{2}^{\prime} \cdots v_{k}^{\prime} v_{i}^{+} v_{i}^{-} v_{1}^{\prime}$ and $v_{k+1}^{\prime} v_{k+2}^{\prime} \cdots v_{p}^{\prime} v_{i} v_{k+1}^{\prime}$ contain vertices from all partite sets of $D$, a contradiction. Analogously, if $v_{k+1}^{\prime} \notin V\left(v_{i}^{-}\right)$and thus $v_{i}^{-} \rightarrow v_{k+1}^{\prime}$, then $v_{1}^{\prime} v_{2}^{\prime} \cdots v_{k}^{\prime} v_{i} v_{i}^{+} v_{1}^{\prime}$ and $v_{k+1}^{\prime} v_{k+2}^{\prime} \cdots v_{p}^{\prime} v_{i}^{-} v_{k+1}^{\prime}$ are weakly complementary cycles of $D$, also a contradiction. Consequently, let $v_{k}^{\prime} \in V\left(v_{i}^{+}\right)$and $v_{k+1}^{\prime} \in V\left(v_{i}^{-}\right)$. If 
$v_{i}^{+} \rightarrow v_{k+1}^{\prime}$, then $D$ contains the cycles $v_{1}^{\prime} v_{2}^{\prime} \cdots v_{k}^{\prime} v_{i} v_{1}^{\prime}$ and $v_{k+1}^{\prime} v_{k+2}^{\prime} \cdots v_{p}^{\prime} v_{i}^{+} v_{k+1}^{\prime}$, a contradiction. If $v_{k}^{\prime} \rightarrow v_{i}^{-}$, then $v_{1}^{\prime} v_{2}^{\prime} \cdots v_{k}^{\prime} v_{i}^{-} v_{1}^{\prime}$ and $v_{k+1}^{\prime} v_{k+2}^{\prime} \cdots v_{p}^{\prime} v_{i} v_{k+1}^{\prime}$ contain vertices from all partite sets of $D$, also a contradiction. Hence let $v_{k+1}^{\prime} \rightarrow v_{i}^{+}$ and $v_{i}^{-} \rightarrow v_{k}^{\prime}$. Now it follows that $D$ contains the cycle $C^{\prime}=v_{i}^{-} v_{k}^{\prime} v_{k+1}^{\prime} v_{i}^{+} v_{i}^{-}$. If $k \neq 2$ or $k \neq p-2$ or $k=2=p-2$ and $v_{1}^{\prime} \notin V\left(v_{p}^{\prime}\right)$, then $C^{\prime}$ and one of the cycles $v_{1}^{\prime} v_{2}^{\prime} \cdots v_{k-1}^{\prime} v_{k+2}^{\prime} v_{k+3}^{\prime} \cdots v_{p}^{\prime} v_{i} v_{1}^{\prime}$ and $v_{1}^{\prime} v_{2}^{\prime} \cdots v_{k-2}^{\prime} v_{k+2}^{\prime} v_{k+3}^{\prime} \cdots v_{p}^{\prime} v_{i} v_{1}^{\prime}$ and $v_{1}^{\prime} v_{2}^{\prime} \cdots v_{k-1}^{\prime} v_{k+3}^{\prime} v_{k+4}^{\prime} \cdots v_{p}^{\prime} v_{i} v_{1}^{\prime}$ are weakly complementary cycles of $D$, a contradiction. Hence let $k=2, p=4$ and $v_{1}^{\prime} \in V\left(v_{4}^{\prime}\right)$. If $\left|D_{m}\right| \geq 2$ with $m \in\{2,3\}$, then $C^{\prime}$ and $v_{1}^{\prime} v_{m}^{\prime \prime} v_{4}^{\prime} v_{i} v_{1}^{\prime}$ contain vertices from all partite sets of $D$, a contradiction. Consequently we have $\left|D_{2}\right|=\left|D_{3}\right|=1$. Now it is obvious that $D-\left\{v_{2}^{\prime}, v_{3}^{\prime}\right\}$ is not strong, a contradiction to $\kappa(D)=3$.

Subcase 3.3. Assume that $v_{m} \leadsto \bigcup_{j=2}^{p-1} D_{j}$ or $\bigcup_{j=2}^{p-1} D_{j} \leadsto v_{m}$ for each $m \in\{1,2,3\}$. Since $D-\left\{v_{1}^{\prime}\right\}$ as well as $D-\left\{v_{p}^{\prime}\right\}$ are strong, we deduce that there are vertices $v_{i}, v_{j} \in V(C)$ with $i \neq j$ such that $v_{i} \rightarrow v_{2}^{\prime}$ and $v_{p-1}^{\prime} \rightarrow v_{j}$, and thus $\left(D_{2} \cup D_{3} \cup\right.$ $\left.\cdots \cup D_{p-1}\right) \leadsto v_{j}$ and $v_{i} \leadsto\left(D_{2} \cup D_{3} \cup \cdots D_{p-1}\right)$. Let $v_{m} \in V(C)-\left\{v_{i}, v_{j}\right\}$.

Suppose that $v_{j} \rightarrow v_{i}$. Then $D$ contains the cycle $C^{\prime}=v_{2}^{\prime} v_{3}^{\prime} \cdots v_{p-1}^{\prime} v_{j} v_{i} v_{2}^{\prime}$. If $v_{1}^{\prime} \notin V\left(v_{p}^{\prime}\right)$, then $D$ contains the weakly complementary cycles $C^{\prime}$ and $v_{1}^{\prime} v_{p}^{\prime} v_{m} v_{1}^{\prime}$, a contradiction. Hence let $v_{1}^{\prime} \in V\left(v_{p}^{\prime}\right)$. If $\left|D_{l}\right| \geq 2$ with $l \in\{2, p-1\}$, then $C^{\prime}$ and $v_{1}^{\prime} v_{l}^{\prime \prime} v_{p}^{\prime} v_{m} v_{1}^{\prime}$ are weakly complementary cycles of $D$, also a contradiction. Consequently, let $\left|D_{2}\right|=\left|D_{p-1}\right|=1$. If $p \geq 5$, then $v_{1}^{\prime} v_{2}^{\prime} v_{p}^{\prime} v_{m} v_{1}^{\prime}$ and $v_{i} v_{3}^{\prime} v_{4}^{\prime} \cdots v_{p-1}^{\prime} v_{j} v_{i}$ or $v_{i} v_{4}^{\prime} v_{5}^{\prime} \cdots v_{p-1}^{\prime} v_{j} v_{i}$ contain vertices from all partite sets of $D$, a contradiction. And if $p=4$, then $D-\left\{v_{2}^{\prime}, v_{3}^{\prime}\right\}$ is not strong, a contradiction to $\kappa(D)=3$.

Consequently, it remains to consider the case that $v_{i} \rightarrow v_{j} \rightarrow v_{m} \rightarrow v_{i}$. If $v_{j} \notin V\left(v_{2}^{\prime}\right)$ and $v_{i} \notin V\left(v_{p-1}^{\prime}\right)$, then $D$ contains the weakly complementary cycles $v_{1}^{\prime} v_{2}^{\prime} v_{j} v_{m} v_{1}^{\prime}$ and $v_{3}^{\prime} v_{4}^{\prime} \cdots v_{p}^{\prime} v_{i} v_{3}^{\prime}$ or $v_{4}^{\prime} v_{5}^{\prime} \cdots v_{p}^{\prime} v_{i} v_{4}^{\prime}$, a contradiction. If $v_{j} \in V\left(v_{2}^{\prime}\right)$ and $v_{i} \in V\left(v_{p-1}^{\prime}\right)$, then it is straightforward to see that $v_{1}^{\prime} v_{p-1}^{\prime} v_{j} v_{m} v_{1}^{\prime}$ and $v_{2}^{\prime} v_{3}^{\prime} \cdots v_{p-2}^{\prime} v_{p}^{\prime} v_{i} v_{2}^{\prime}$ or $v_{2}^{\prime} v_{3}^{\prime} \cdots v_{p-3}^{\prime} v_{p}^{\prime} v_{i} v_{2}^{\prime}$ are two cycles that contain vertices from all partite sets of $D$, also a contradiction. Hence let $v_{j} \in V\left(v_{2}^{\prime}\right)$ and $v_{i} \rightarrow v_{p-1}^{\prime}$. If $v_{2}^{\prime} \rightarrow v_{m}$, then $D$ contains the weakly complementary cycles $v_{i} v_{2}^{\prime} v_{m} v_{i}$ and $v_{1}^{\prime} v_{3}^{\prime} v_{4}^{\prime} \cdots v_{p}^{\prime} v_{j} v_{1}^{\prime}$ or $v_{1}^{\prime} v_{4}^{\prime} v_{5}^{\prime} \cdots v_{p}^{\prime} v_{j} v_{1}^{\prime}$, a contradiction. Consequently, let $v_{m} \rightarrow v_{2}^{\prime}$. Now, we have two vertices $v_{j}, v_{m} \in V(C)$ such that $v_{m} \rightarrow v_{2}^{\prime}, v_{p-1}^{\prime} \rightarrow v_{j}$ and $v_{j} \rightarrow v_{m}$. As above we arrive at a contradiction. Finally if $v_{2}^{\prime} \rightarrow v_{j}$ and $v_{i} \in V\left(v_{p-1}^{\prime}\right)$, then observing the converse $D^{-1}$ of $D$, we also arrive at a contradiction. This completes the proof of this theorem.

A direct consequence of this theorem is the following result.

Corollary 3.2. Every 3-strong c-partite tournament with $c \geq 3$ and at least 8 vertices is weakly cycle complementary.

The following example presents two 2-strong $c$-partite tournaments with $c \geq 3$ and at least 6 vertices that even do not contain two vertex disjoint cycles, and thus no weakly complementary cycles. 
Example 3.3. The 4-partite tournament $D_{1}$ and the 3-partite tournament $D_{2}$ of Figure 3 are 2-strong and do not have any two vertex disjoint cycles. Thus they do not have two vertex disjoint cycles with vertices from all partite sets, which means that $D_{1}$ and $D_{2}$ are not weakly cycle complementary.

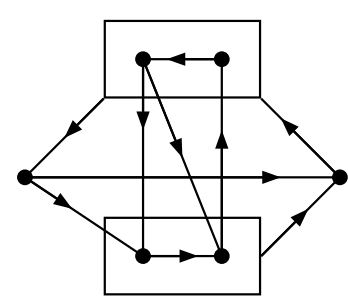

$D_{1}$

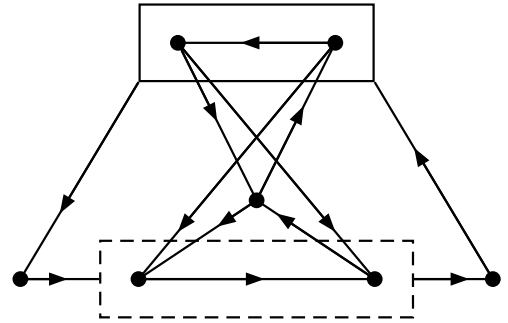

$D_{2}$

Figure 3: The 2-strong multipartite tournaments $D_{1}$ and $D_{2}$ without a pair of vertex disjoint cycles.

Since the authors found some more 2-strong multipartite tournaments with this property it may become difficult to characterize all 2-strong multipartite tournaments that are weakly cycle complementary. Nevertheless it would be interesting to solve this problem.

Problem 3.4. Characterize all 2-strong $c$-partite tournaments $D$ with $c \geq 3$ and $|V(D)| \geq 6$ that are weakly cycle complementary.

\section{References}

[1] J. A. Bondy, Disconnected orientation and a conjecture of Las Vergnas, J. London Math. Soc., 14(1976), 277-282.

[2] W. D. Goddard and O. R. Oellermann, On the cycle structure of multipartite tournaments, in: Y. Alavi, G. Chartrand, O.R. Oellermann, A.J. Schenk (Eds.), Graph Theory Combinat. Appl., 1, Wiley Interscience, New York (1991), 525-533.

[3] T. Korneffel, D. Meierling, L. Volkmann and S. Winzen, Complementary cycles in regular multipartite tournaments, where one cycle has length five, submitted.

[4] K. B. Reid, Two complementary circuits in two-connected tournaments, Ann. Discrete Math., 27(1985), 321-334. 
[5] Z. Song, Complementary cycles of all length in tournaments, J. Combin. Theory Ser. B, 57(1993), 18-25.

[6] M. Tewes and L. Volkmann, Vertex deletion and cycles in multipartite tournaments, Discrete Math., 197-198(1999), 769-779.

[7] L. Volkmann, Cycles in multipartite tournaments: results and problems, Discrete Math., 245(2002), 19-53.

[8] L. Volkmann, All regular multipartite tournaments that are cycle complementary, Discrete Math., 281(2004), 255-266.

[9] L. Volkmann, Complementary cycles in regular multipartite tournaments, where one cycle has length four, Kyungpook Math. J., 44(2004), 219-247.

[10] L. Volkmann, Complementary cycles in regular multipartite tournaments, Australas. J. Combin., 31(2005), 119-134.

[11] A. Yeo, Diregular c-partite tournaments are vertex-pancyclic when $c \geq 5$, J. Graph theory, 32(1999), 137-152. 